Mens

Revue d'histoire intellectuelle et culturelle

mens

\title{
Portrait de groupe des " jeunes gens " en lecteurs, en écrivains et en professeurs (1830-1850)
}

\section{Micheline Cambron}

Volume 19, numéro 1-2, automne 2018, printemps 2019

Les années 1840 : rupture ou réarticulation des possibles ?

URI : https://id.erudit.org/iderudit/1070069ar

DOI : https://doi.org/10.7202/1070069ar

Aller au sommaire du numéro

Éditeur(s)

Centre de recherche en civilisation canadienne-française

ISSN

1492-8647 (imprimé)

1927-9299 (numérique)

Découvrir la revue

Citer cet article

Cambron, M. (2018). Portrait de groupe des « jeunes gens » en lecteurs, en écrivains et en professeurs (1830-1850). Mens, 19(1-2), 63-94.

https://doi.org/10.7202/1070069ar
Résumé de l'article

Les années 1840 ne sont pas seulement celles qui suivent les rébellions, elles sont marquées par de nombreuses initiatives liées à la formation de la jeunesse. Les journaux à bas prix de François-Xavier Garneau (L'Abeille canadienne, L'Institut), ceux de James Huston (L'Artisan), de Napoléon Aubin (Le Castor) et de Louis-Octave LeTourneux (La Revue canadienne) accompagnent la mise en place d'associations qui font de l'éducation, du perfectionnement des jeunes gens leur objectif cardinal. Ces jeunes gens sont ceux qui ont, dès les années 1820 et surtout 1830, profité de la mise en oeuvre d'une stratégie de scolarisation pour tous dans le Bas-Canada, laquelle se déploie dans les écoles d'enseignement mutuel et dans les écoles de syndics. Parallèlement naissent les premiers gestes médiatiques appelant la création d'une littérature canadienne et les premières oeuvres qui explicitement s'en réclament. Dans ce mouvement général, les jeunes gens se font tour à tour, et les uns pour les autres, lecteurs, écrivains et professeurs. Durant près de deux décennies, une vision mutualiste de l'éducation traverse le discours culturel, invite à la construction d'un espace public dynamique et soutient, grâce à des figures et à des oeuvres phares, le désir de créer une culture nationale. 


\title{
Portrait de groupe des «jeunes gens» en lecteurs, en écrivains et en professeurs $(1830-1850)^{1}$
}

\author{
Micheline Cambron \\ Université de Montréal et CRILCQ
}

À Yvan Lamonde

\section{Résumé}

Les années 1840 ne sont pas seulement celles qui suivent les rébellions, elles sont marquées par de nombreuses initiatives liées à la formation de la jeunesse. Les journaux à bas prix de François-Xavier Garneau (L'Abeille canadienne, L'Institut), ceux de James Huston (L'Artisan), de Napoléon Aubin (Le Castor) et de Louis-Octave LeTourneux (La Revue canadienne) accompagnent la mise en place d'associations qui font de l'éducation, du perfectionnement des jeunes gens leur objectif cardinal. Ces jeunes gens sont ceux qui ont, dès les années 1820 et surtout 1830, profité de la mise en œuvre d'une stratégie de scolarisation pour tous dans le Bas-Canada, laquelle se déploie dans les écoles d'enseignement mutuel et dans les écoles de syndics. Parallèlement naissent les premiers gestes médiatiques appelant la création d'une littérature canadienne et les premières œuvres qui explicitement s'en réclament. Dans ce mouvement général, les jeunes gens se font tour à tour, et les uns pour

1 Je remercie le CRSH et le FRQ dont les subventions des deux dernières décennies m'ont permis de rassembler les matériaux à l'origine de la présente réflexion. Je remercie également les nombreux assistants de recherche et les étudiants qui ont soutenu et stimulé ma réflexion et Mathilde Cambron-Goulet pour ses commentaires éclairés. 
les autres, lecteurs, écrivains et professeurs. Durant près de deux décennies, une vision mutualiste de l'éducation traverse le discours culturel, invite à la construction d'un espace public dynamique et soutient, grâce à des figures et à des œuvres phares, le désir de créer une culture nationale.

\section{Abstract}

The decade of 1840 is not only the one following the rebellions, it is also characterized by numerous actions dedicated to educational development of the youth. The low-cost periodics of François-Xavier Garneau (L'Abeille canadienne, L'Institut), those of James Huston (L'Artisan) and Napoléon Aubin (Le Castor) and, in a way, Revue canadienne of Louis-Octave LeTourneux, must be viewed in that perspective. All those go side-by-side with the creation, in Quebec and Montreal, of associations aimed to a reciprocal education of young and less young men, seen as the base of intellectual fulfilment. Those young men had the opportunity to benefit of the establishment of new educational grounds in the Bas-Canada: peer learning (Lancastrian) schools and syndics schools. Simultaneously, medias begin to encourage the emergence of a French Canadian literature and the first important pieces are published. In that general movement, the young men act as readers, writers and teachers. During the decades of 1830 and 1840, a strong commitment in education by the peers ran through the social discourse, contributed to enlarge and vitalize the participation of the youth in public space and sustained, thanks to major actors and flagship productions, the desire to create a national French culture in Canada.

Les décennies 1830 et 1840 sont marquées, au Québec, par de nombreuses initiatives liées à la formation de la jeunesse. Les journaux à tarif réduit de François-Xavier Garneau précèdent ou accompagnent la mise en place d'associations qui feront de l'instruction que se prodiguent mutuellement des jeunes gens leur objectif cardinal. La mémoire commune a retenu le nom de l'Institut canadien de 
Montréal, fondé en $1844^{2}$, mais il faudrait aussi parler de la Société canadienne d'études littéraires et scientifiques, fondée en 1843, de la Société de discussion de Québec, fondée en 1843, de la Société des amis, fondée à Montréal en $1844^{3}$, et de toutes sortes d'initiatives plus modestes ${ }^{4}$. La multiplication à Québec, et ensuite à Montréal, de "cours d'instruction populaire» et de "lectures» organisés par diverses instances semble s'inscrire dans un mouvement général d'enthousiasme à l'égard de l'acquisition de connaissances, dont on peut croire qu'il a été préparé par une alphabétisation issue de la fondation d'écoles, gratuites ${ }^{5}$ ou non, privées, de fabriques ou de syndics, et par plus d'une décennie de discussions publiques sur l'éducation

2 Grâce aux travaux d'Yvan Lamonde, en particulier Gens de parole: conférences publiques, essais et débats à l'Institut canadien de Montréal 1845-1871, Montréal, Boréal, 1990. L'Institut est fondé en 1844, et la première séance publique a lieu en janvier 1845 .

3 Fondée par Lactance Papineau en novembre 1844, cette association prend le relais de la Société littéraire, fondée en 1837 par son frère Amédée Papineau. Yvan Lamonde et Sophie Montreuil (dir.), Lire au Québec au XIX siècle, Montréal, Éditions Fides, 2003, p. 99.

4 La lecture de L'Artisan et du Castor permet, pour les années 1842-1845, de découvrir de nombreuses activités «instructives», liées à Québec à des associations de divers types.

5 Certaines entreprises sont d'envergure, comme celle de la Société d'éducation du district de Québec, présidée par Joseph-François Perrault, fondée en 1821. Cette société rassemblait plusieurs notables de Québec préoccupés par l'éducation. Il s'agissait de scolariser gratuitement les enfants pauvres de la ville, dont le nombre était estimé à 1000 . Une école fut ouverte «avec 90 enfants et [...] allait en compter 415 en 1833, filles et garçons, Canadiens et Irlandais» et celle de la Société d'école britannique et canadienne de Québec, fondée en 1823, également par Perrault, sans enseignement religieux autre que la lecture de la Bible, et qui aurait reçu, de sa fondation à 1837, 2360 enfants des deux sexes et préparé 46 maîtres capables d'enseigner d'après le système de Joseph Lancaster. Perrault fonda trois autres écoles, une de garçons et une de filles au faubourg Saint-Louis, en 1830 et, vraisemblablement, entre 1830 et 1833, une école pratique d'agriculture sur les bords de la rivière Saint-Charles en 1832. Ces informations sont tirées de Claude Galarneau, "PERRAULT, JOSEPHFRANÇOIS ", Dictionnaire biographique du Canada, vol. 7, Québec, Université Laval; Toronto, University of Toronto, 2003, [En ligne], [http://www.biographi. ca/fr/bio/perrault_joseph_francois_7F.html] (10 mars 2017). 
précédant et entourant la promulgation de la Loi des écoles de syndics (1829-1836) ${ }^{6}$. Il faut remarquer que, dans le cadre des associations nouvelles qui se mettent en place à partir de la fin des années 1830 , le désir d'apprendre est capital (cours, conférences, essais, débats). L'engouement lié à la prise de parole et aux discussions qui en découlent est perceptible dans les journaux qui rendent compte de ces activités, trop souvent réduites dans notre mémoire à celles de l'Institut canadien de Montréal. Outre le fait que cela élude leur importance dans la ville de Québec, cela empêche de comprendre l'évolution d'un certain rapport au savoir, marqué par la foi dans la gratuité scolaire, dans les vertus de l'autonomie intellectuelle et en l'émancipation démocratique ${ }^{7}$. Les travaux récents de Louise Bienvenue, d'Ollivier Hubert et de Christine Hudon, de même que ceux de Jean-Pierre Proulx, de Christian Dessureault et de Paul Aubin jettent un éclairage nouveau sur les pratiques éducatives concrètes qui modelèrent ceux que les périodiques désignent comme les «jeunes gens ${ }^{8}$ ». La lecture des périodiques qui leur sont explicitement destinés permet aussi de mieux comprendre leurs activités et leurs projets. Nous nous appuierons donc ici sur l'analyse de divers types de discours, depuis les prospectus qui rendent explicites les objectifs poursuivis par les journaux

6 Voir Jean-Pierre Proulx, avec la collaboration de Christian Dessureault et de Paul Aubin, La genèse de l'école publique et de la démocratie scolaire au Québec: les écoles de syndics 1814-1838, Québec, Presses de l'Université Laval, 2014, en particulier p. 139-182.

7 Ces mouvements sont aussi présents en France, et de récents travaux leur ont été consacrés. Pour un état de ces travaux, voir Emmanuel Fureix et François Jarrige, "Vers une culture démocratique?" dans La modernité désenchantée: relire l'histoire du XIXe siècle français, Paris, La Découverte, 2015, p. 156-165.

8 Louise Bienvenue, Ollivier Hubert et Christine Hudon, Le collège classique pour garçons: études historiques sur une institution québécoise disparue, Montréal, Éditions Fides, 2014; Proulx et al., La genèse de l'école publique et de la démocratie scolaire au Québec. Sur les pratiques pédagogiques de l'enseignement simultané, voir Paul Aubin, "La formation à l'enseignement dans une communauté enseignante: les Frères des écoles chrétiennes du Québec 1837-1980", Historical Studies in Education = Revue d'histoire de l'éducation, vol. 28, $\mathrm{n}^{\circ} 2$ (automne 2016), p. 30-31, [En ligne], [http://historicalstudiesineducation.ca/ index.php/edu_hse-rhe/article/view/4494/4729] (9 mars 2017). 
destinés aux «jeunes gens» jusqu’à des extraits de textes littéraires, afin d'approfondir ce portrait de groupe qui vise à mettre en lumière l'intrication de la conception du savoir qui est la leur et du premier mouvement coordonné de création d'une littérature nationale.

\section{Des «jeunes gens»}

Attardons-nous d'abord à ces «jeunes gens» dont la figure traverse de toutes sortes de manières le discours culturel de l'époque. C'est à eux que s'adresse l'appel «À la jeunesse canadienne» du Populaire, désireux, en 1837, de guider les jeunes écrivains ${ }^{9}$. C'est aussi eux qui sont mis en scène, d'une manière toute balzacienne, dans Linfluence d'un livre ${ }^{10}$ et qui, au théâtre, dans les pièces de Pierre Petitclair ${ }^{11}$, triomphent des vieux barbons et de leur naïveté. Ce sont eux, les jeunes écrivains canadiens méprisés, que Joseph Doutre défend dans la préface de son roman Les fiancés de $1812^{12}$; eux encore qui seront interpellés par la Revue canadienne ${ }^{13}$ et qui y écriront. À eux enfin,

9 "À la jeunesse canadienne», Le Populaire, Montréal, 10 avril 1837. Cet appel est reproduit dans Le Télégraphe = The Telegraph, Québec, 14 avril 1837. Ce bihebdomadaire a été fondé par Philippe Aubert de Gaspé fils et Napoléon Aubin.

${ }^{10}$ Dans les chapitres «L'Autopsie» et "Un jeune médecin» (Philippe Aubert de Gaspé fils, L'influence d'un livre, Boréal compact, 1996, p. 65-[72] et 107-[113] ; première édition, Québec, William Cowan et fils, 1837).

${ }^{11}$ Pierre Petitclair, Griphon, ou la Vengeance d'un valet, comédie en trois actes, Québec, William Cowan, imprimeur, 1837; La donation, créée le 16 novembre 1842, publiée dans L'Artisan (15, 22, 26, 29 décembre 1842). Il n'existe pas d'édition contemporaine outre la traduction parue dans Leonard E. Doucette, The Drama of Our Past: Major Plays from Nineteenth-Century Quebec, Toronto, University of Toronto Press, 1997.

12 «Nous nous consolerons volontiers des critiques si l'humilité de notre nom peut faire comprendre à nos jeunes amis qu'ils sont plus capables qu'ils ne le pensent ", écrit Doutre, après avoir défendu les tentatives littéraires des jeunes Canadiens contre les prétentions des faux Parisiens pleins de fatuité et contre les vieillards «à qui l'âge n'a pas laissé de dents [pour] goûter les fruits dont la dureté fait l'envie des jeunes mâchoires" (Joseph Doutre, Les fiancés de 1812, Montréal, Louis Perrault, imprimeur, 1844, p. xx).

13 "Nous fesons $[s i c]$ surtout un appel à nos jeunes compatriotes canadiens, de toutes professions, de tous états, de toutes conditions; ce journal est le leur" (Louis-O. LeTourneux, "Prospectus», Revue canadienne). Le texte est daté du 
tout au moins à ceux qui sont "studieux", que sera d'abord destiné Le répertoire national, en $1848^{14}$. Ils s'y retrouveront d'ailleurs nombreux à titre de signataires de pièces diverses ${ }^{15}$.

Qui sont ces "jeunes gens"? Disons que ce sont des jeunes hommes encore en formation. Le terme revient de manière récurrente dans les textes de l'époque. L'une des associations nouvelles a même porté, durant quelques mois, le nom de Société littéraire des jeunes gens avant de devenir la Société canadienne d'études littéraires et scientifiques. Marc Lebel évalue que l'âge médian des 21 fondateurs de la Société dont nous connaissons la date de naissance se situe autour de 20 ans ${ }^{16}$; Il insiste aussi sur la diversité de leur profil (ils sont professionnels, artisans ou ouvriers), frappé qu'il est, et avec raison, par les écarts sociaux que ces sociétés semblent vouloir gommer, ce qui témoigne de la mission égalitariste et démocratique portée, selon eux, par l'éducation ${ }^{17}$. Lamonde insiste,

14 décembre 1844 et jouxte un texte assez différent en anglais, également signé par LeTourneux, qui est propriétaire et rédacteur du périodique. Ce premier numéro porte, après la page couverture, un texte intitulé «Aux membres de la Société des amis", qui insiste sur la mission éducative de la société, dont les membres sont tendus vers «le grand but de notre perfectionnement moral et intellectuel».

${ }^{14}$ Le répertoire national ou Recueil de littérature canadienne est destiné aux jeunes gens studieux, aux écrivains canadiens et aux amoureux de la littérature nationale. Voir James Huston, Prospectus, Québec, Le Canadien, 29 octobre 1847 et «Introduction", Le répertoire national ou Recueil de littérature canadienne, compilé et publié par James Huston, membre de l'Institut canadien de Montréal, Montréal, Imprimerie Lovell et Gibson, 1848-1850, p. III. Voir, à ce propos, Micheline Cambron, "Place aux textes!": littérature québécoise et injonctions de lecture» (à paraitre).

${ }^{15}$ David Hayne fait remarquer que les récits du Répertoire national dont les auteurs sont identifiables "appartiennent à une même génération, c'est-à-dire que tous sont nés autour de 1820, plus précisément entre 1812 et 1825" ("L'influence des auteurs français sur les récits de 1820 à 1845 ", dans Maurice Lemire (dir.), Le romantisme au Canada, Québec, Nuit blanche éditeur, p. 45, coll. "Les Cahiers du CRELIQ").

${ }^{16}$ Marc Lebel, «François-Xavier Garneau et la Société de discussion de Québec», dans Gilles Gallichan, Kenneth Landry et Denis Saint-Jacques (dir.), FrançoisXavier Garneau, une figure nationale, Québec, Éditions Nota bene, 1998, p. 100.

17 "Ses membres [ceux de la Société] se réunissent tous les samedi pour discuter "de littérature, de sciences, d'arts, de morale, de politique". Composée de "jeunes 
quant à lui, sur le jeune âge auquel les membres de l'Institut canadien prennent la parole pour la première fois ${ }^{18}$. Il s'appuie sur des textes de Joseph Doutre, qu'il considère comme le "chroniqueur le plus sensible de cette génération» et suggère que les activités de l'Institut canadien seraient l'affaire des «jeunes gens" marqués par l'impasse politique et culturelle de $1840^{19}$. La désignation générale de ces «jeunes gens» comme professionnels ou "jeunes bourgeois», par Fernand Dumont, entre autres, n'est évidemment pas fausse, mais elle efface, sinon les origines concrètes de ces jeunes gens, - sur lesquelles Dumont, pourtant, insiste: ils sont «[i] ssus d'un milieu populaire très particulier ${ }^{20} »-\mathrm{du}$ moins le rapport singulier à la connaissance qui est le leur. Ces jeunes gens, attachés à la mentalité libérale, pour lesquels «l'instruction mutuelle est un capital, le savoir, un avoir ${ }^{21}$ » et qui feront du tournant de la décennie 1840 une période particulièrement riche sur le plan littéraire, ont un rapport singulier à la connaissance.

Insistons d'abord sur la nature de leurs formations respectives. Il ne suffit pas, en effet, de les rattacher à un collège pour pouvoir les fondre en une seule classe sociale. Plusieurs sont allés au collège, mais nous ne savons pas toujours bien ce que cela recouvre car il est manifestement erroné de croire que la formation était la même pour tous. Nous avons tendance à projeter sur les parcours académiques et intellectuels de ces jeunes gens les catégories qui sont les nôtres et à leur imaginer des cursus identiques. Ce n'était manifestement

gens de toutes les classes, de toutes les professions et métiers", "d'étudiants en droit, en médecine, d'artistes et d'artisans", elle "cherche à répandre le goût de la discussion utile parmi la jeunesse et la classe ouvrière" ", Ibid., p. 95. Lebel tire ses citations d'articles de journaux parus dans Le Canadien, L'Artisan et Le Fantasque, en mars 1843 (Ibid., note 26, p. 149).

${ }^{18}$ Lamonde, Gens de parole, p. 48.

${ }^{19}$ Ibid., p. 29-33.

${ }^{20}$ Fernand Dumont, "Idéologie et conscience historique dans la société canadienne-française du XIX ${ }^{e}$ siècle», dans Jean-Paul Bernard (dir.), Les idéologies québécoises au $19^{\circ}$ siècle, Montréal, Les éditions du Boréal express, 1973, p. 61-82.

${ }^{21}$ Lamonde, Gens de parole, p. 28. 
pas le cas. Né en 1824, Pierre-Joseph-Olivier Chauveau est en $4^{\mathrm{e}}$ en 1831-1832; au même moment, François-Réal Angers, né en 1812, fait sa classe de rhétorique $\left(2^{\mathrm{e}}\right)$. Autre exemple, Pierre Petitclair, sorte de whiz kid du Petit Séminaire, quitte le collège en début de troisième classe (celle de rhétorique), mais n'aura été étudiant que quatre années, ayant fait cinq années en quatre ${ }^{22}$. Bien que plus jeune de quatre ans que Garneau, il entrera comme lui dans la vie active en $1830^{23}$. D'où tient-il la formation préalable qui lui a permis une telle progression? Comme Garneau, il a fréquenté une école de Joseph-François Perrault, peut-être au même moment. C'est dire que cette école pratiquant l'enseignement mutuel a sans doute pesé considérablement dans son éducation. Perrault fut un ardent défenseur de la méthode d'enseignement mutuel de Joseph Lancaster, qu'il pratiqua en y apportant "des modifications avantageuses", selon le journal Le Canadien du 30 septembre $1833^{24}$. Cet enseignement, qui rompt avec celui qui, traditionnellement, repose sur le face à face entre l'élève et le maître, vise de grands groupes et mise sur l'enseignement par les pairs. Il jouit alors d'un grand prestige $^{25}$.

${ }^{22}$ Fiche d'élève de Pierre Petitclair, Liste des élèves du Petit Séminaire (Manuscrit [cahier] $n^{\circ} 5$ et Ordo Locorum, Fonds du Petit Séminaire de Québec [Musée de l'Amérique française]. Voir aussi Noël Baillargeon, Histoire du Séminaire de Québec de 1800 à 1850, Sainte-Foy, Presses de l'Université Laval, 1994, p. 279-280. Pour une biographie mise à jour de Pierre Petitclair, voir Micheline Cambron et Louise Frappier, "Pierre Petitclair, la vie et l'œuvre d'un écrivain invisible", dans Pierre Petitclair, Une partie de campagne: comédie en deux actes, texte établi et annoté par Micheline Cambron et Louise Frappier, avec la collaboration de Mathilde Cambron-Goulet, Montréal, Alias [groupe Nota bene], 2017, p. 137-153.

${ }^{23}$ Tous deux seront des employés d'Archibald Campbell, lequel est aussi membre de la Société d'éducation de Québec. On trouve la liste des membres fondateurs de la Société, dans Proulx et al., La genèse de l'école publique et de la démocratie scolaire au Québec, p. 67.

${ }^{24}$ Ibid., p. 334.

${ }^{25}$ Sur l'enseignement mutuel, voir les travaux de Christian Nique, dont Comment l'école devint une affaire d'État (1815-1840), Paris, Nathan, 1990, et l'article, plus récent, de Sylvie Jouan: «Enseignement mutuel et enseignement simultané: 
Nous savons désormais, grâce aux travaux de Bienvenue, de Hubert et de Hudon, qu'une formation dans un collège pouvait revêtir des formes très variées, que la mixité des âges, des classes sociales et des cursus était la règle plutôt que l'exception ${ }^{26}$. Certains programmes, commerciaux, industriels, agricoles, commencent à susciter l'intérêt des chercheurs. D'autres initiatives demeurent encore mystérieuses, comme les classes lancastriennes du Petit Séminaire de Québec (1830-1837) mises en œuvre par Jean Holmes, sous la responsabilité de l'abbé J.-F. Baillargé, et dont les listes de 1830-1831 et de 1831-1832 (respectivement 103 et 93 inscrits) ont été conservées dans les archives du Séminaire même si l'enseignement mutuel a été interdit par le pape Léon XII en $1824^{27}$ (!) (figure 1). Chose certaine, la formation variait selon les collèges, et le latin ne constituait pas une matière universellement enseignée ${ }^{28}$. De plus, la plupart des étudiants qui suivaient la filière latine ne terminaient pas le programme entier du ratio studiorum. La cléricature, par exemple, ne le requérait aucunement, comme le prouve le statut de notaire de François-Xavier Garneau, qui n'avait pas du tout fréquenté le collège ${ }^{29}$.

quelle conception de l'apprentissage se cache derrière le choix pédagogique du mode simultané par le Ministère Guizot en France?», 2015, [En ligne], [www. meirieu.com/PATRIMOINE/mutuel_simultane_jouan.pdf] (9 mars 2017).

${ }^{26}$ Bienvenue, Hubert et Hudon parlent pour les collèges d'un éventail de formations, par exemple, de filières commerciales et industrielles. Les variables qui segmentent la population des collèges peuvent être visualisées dans les nombreux tableaux du chapitre d'Ollivier Hubert, «Le Collège de Montréal et ses clientèles au xiX ${ }^{\mathrm{e}}$ siècle: multiplicité des attentes et pluralité de l'offre», dans Bienvenue, Hubert et Hudon, Le collège classique pour garçons, p. 55-87.

${ }^{27}$ Liste manuscrite des élèves du Séminaire pour 1829-1830, Fonds du Petit Séminaire de Québec. Si l'on ajoute ces élèves à ceux des trois écoles de Perrault, respectivement fondées en 1821, en 1823 et en 1830, la région de Québec aurait compté en 1829-1830 au moins 400 élèves instruits selon la méthode d'enseignement mutuel.

${ }^{28}$ Hubert, "Le collège de Montréal et ses clientèles au XIX ${ }^{\mathrm{e}}$ siècle», p. 72-75.

${ }^{29}$ Les biographies sont concordantes sur ce point (Pierre Savard et Paul Wyczynski, "GARNEAU, FRANÇOIS-XAVIER», Dictionnaire biographique du Canada, vol. 9, Québec, Université Laval; Toronto, University of Toronto, 1977, [En ligne], 


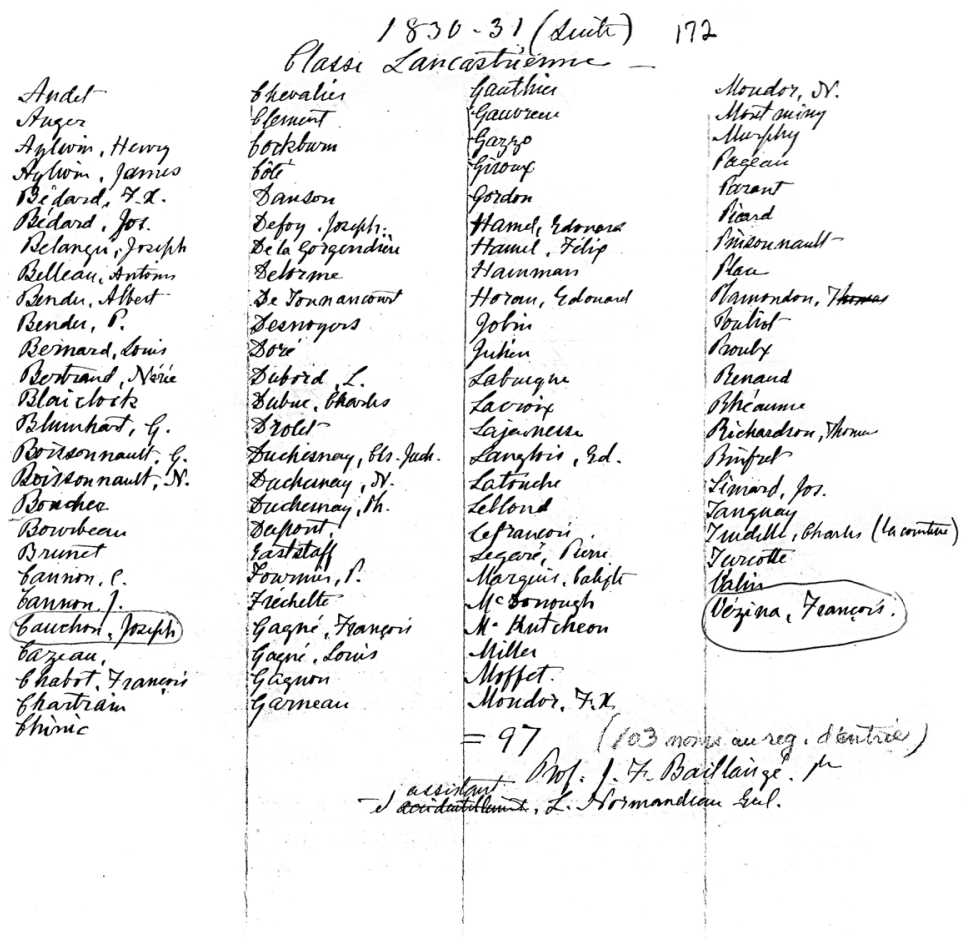

Figure I - Liste d'élèves du Petit Séminaire de Québec (1765-1849) par Amédée-E. Gosselin, 1916 (manuscrit), 1830-1831 (suite) Classe Lancastrienne, [p.] 172.

La fréquentation d'un collège ne garantit donc pas une formation qui serait la même pour tous. En outre, et la formation primaire reçue semble jouer un rôle déterminant. Ceux que l'on désigne généralement comme autodidactes, et qui seront parfois dénigrés pour cela ${ }^{30}$, ont pu bénéficier, à Québec, d'une formation

[http://www.biographi.ca/fr/bio/garneau_francois_xavier_9F.html] (10 mars 2017). En effet, F.-X. Garneau n'apparaît pas dans les listes du Petit Séminaire entre 1825 et 1830. Toutefois, un élève de la classe lancastrienne de 1930-1931 se nomme Garneau [sans prénom].

${ }^{30}$ Pensons à l'historien F.-X. Garneau dont l'adhésion aux principes des Lumières viendrait de sa formation non classique, selon ses détracteurs. 
plus ou moins longue dans une des écoles d'enseignement mutuel de Joseph-François Perrault ou, à Montréal, de l'enseignement d'une des nombreuses «écoles privées dirigées par des laïcs dans lesquelles on enseigne surtout en anglais ${ }^{31}$ ». Les plus jeunes pourront avoir eu accès à l'enseignement dispensé dans une des écoles de fabriques ou de syndics, collectivement portées par une "volonté ferme de généraliser l'éducation ${ }^{32}$ ", à partir de 1829. Il est aussi loisible à tous de s'appuyer pour progresser sur l'aide de proches et sur la lecture de manuels ou de journaux faisant la promotion de l'apprentissage autonome, lequel peut alors s'appliquer autant à la lecture qu’à la chimie $^{33}$. Certains ont également eu un accès privilégié à des bibliothèques privées ${ }^{34}$. La variété des parcours, fortement individualisés, rend malaisée toute définition précise d'une classe d'âge à laquelle renverrait l'expression «jeunes gens».

${ }^{31}$ Voir Andrée Dufour, «Diversité institutionnelle et fréquentation scolaire dans l'île de Montréal, en 1825-1835", Revue d'histoire de l'Amérique française, vol. 41, $\mathrm{n}^{\circ} 4$ (1988), p. 507-536. Cité par Proulx, dans La genèse de l'école publique et de la démocratie scolaire au Québec, p. 7.

${ }^{32}$ Mélanie Bédard, "Compte rendu de Jean-Pierre Proulx, avec la collaboration de Christian Dessureault et Paul Aubin, La genèse de l'école publique et de la démocratie scolaire au Québec: les écoles de syndics 1814-1838", Recherches sociographiques, vol. LVI, n 2-3 (mai-décembre 2015), p. 543.

${ }^{33}$ Le Glaneur (journal fondé par J.-B. Boucher-Belleville) en offre des exemples. Dans un article intitulé "Éducation. Enseignement par soi-même», E. B. présente une méthode d'apprentissage autonome de la lecture pour adultes: «Dès qu'on est parvenu, par exemple à Calypso étonnée et attendrie [dans le Télémaque] l'élève sait d'ordinaire lire suffisamment pour déchiffrer des livres, en rapportant ce qu'il ignore à ce qu'il a appris", décembre 1836 (le soulignement est de l'auteur). Une série d'articles pour «ceux qui veulent pratiquer l'agriculture comme un art" (Le Glaneur, avril 1836), tirée par Jean-Baptiste Meilleur de son Cours abrégé de chimie (1833), vise aussi à vulgariser la chimie: "Extraits du cours abrégé de leçon de chimie ", Le Glaneur, numéros d'avril, mai, juin et septembre 1836.

${ }^{34}$ Par exemple, Garneau aurait eu accès à la bibliothèque d'Archibald Campbell et Philippe Aubert de Gaspé fils, qui n'a fait qu'un bref "passage attesté au Séminaire de Nicolet, de 1827 à 1829", selon la "Chronologie» de Rainier Grutman (Philippe Aubert de Gaspé, fils, L'influence d'un livre, Montréal, Boréal compact, 1996, p. 139), aurait donc surtout fréquenté la bibliothèque de son père. 
Il n'est guère plus facile de circonscrire le groupe en croisant formation et occupation. Par exemple, les typographes, considérés comme des artisans, reçoivent, si l'on excepte la nécessité d'un apprentissage en imprimerie, une formation très variée. Joseph Savard a suivi le programme régulier de six années au Petit Séminaire, tandis que d'autres typographes sont des autodidactes déclarés, comme Stanislas Drapeau et James Huston ${ }^{35}$. Napoléon Aubin, typographe et imprimeur, a suivi à Genève une formation expérimentale ${ }^{36}$. Par ailleurs, les typographes constituent un réseau particulièrement vivant: ils fondent à Québec la première association de travailleurs au Canada dès 1836, la Société typographique canadienne, avec «le double but d'être à la fois une société de bienveillance fraternelle et une association d'ouvriers de bonne volonté cherchant mutuellement à s'instruire ${ }^{37}$ \%. Plusieurs typographes sont aussi écrivains ou artistes. Ainsi Napoléon Aubin

${ }^{35}$ Huston et Drapeau seront très actifs dans les associations. Tous deux ont commencé leur vie professionnelle comme apprentis-typographes et, sans doute, petits gazetiers au journal Le Fantasque de Napoléon Aubin. Ils deviendront d'importants éditeurs.

${ }^{36}$ Il est probable que Napoléon [Nicolas-Aimé] Aubin, qui vient de Chêne-Bougeries, tout près de Genève, ait suivi au primaire une formation de type lancastrien. $\mathrm{La}$ Société économique était responsable de l'éducation dans le canton de Genève durant la période de formation d'Aubin. Elle semble s'être tournée vers l'enseignement mutuel dès 1820. Dans le rapport annuel de 1821, il est question des frais de construction d'écoles selon la "nouvelle méthode» et de la création d'une "école lancastrienne» derrière le "collège» (Archives d'État du canton de Genève, section 8, Archives de la Société économique, E1). En 1822, le rapport fait état de désordres commis par les élèves à l'école lancastrienne de Chêne (sans doute Chêne-Bougeries). Aubin termine ses études dans un "collège» près de Genève, sous la responsabilité de la Société économique de Genève, où le programme comportait savoirs techniques et musique (Archives d'État, Recensement, G2 cahier 57, folio 228). Il s'agit possiblement de l'École industrielle de la Société des Arts, qui aurait alors été désignée simplement comme "collège» dans le recensement où apparaît Aubin. L'histoire de l'enseignement à Genève durant les années 1820 est marquée par des polémiques et des expérimentations (I.-A. Verchères, "Premiers projets de réforme 1814-1833", Histoire du collège de Genève, publiée sous les auspices du Département de l'Instruction publique, 1896, p. [281]-297).

37 Voir Éric Leroux, "Culture ouvrière et métiers du livre: la Société typographique de Québec, 1836-1872", Papers of the Bibliographical Society of Canada, vol. 42, $\mathrm{n}^{\circ} 2$ (2004), p. [25]-56. 
est flâneur, poète, prosateur, homme de théâtre, musicien, graveur et chimiste $^{38}$, alors que Joseph Savard fut le plus important metteur en scène à Québec au XIX ${ }^{\mathrm{e}}$ siècle $^{39}$ (figure 2). Leur présence active dans de nombreuses associations dont la Société Saint-Jean-Baptiste et la Société de discussion de Québec, au côté des professionnels que sont les avocats, les notaires et les médecins, témoigne à la fois du désir très vif d'implication dans la collectivité de la part d'un groupe particulièrement sensible aux enjeux de l'alphabétisation, du désir explicite des associations de réduire les fractures sociales et de la solidarité d'une jeunesse liée par des convictions communes, par-delà les lignes de parti. On remarque aussi le rôle central que jouent alors dans la communauté ceux qui participent de près à la vie médiatique, que ce soit à titre de "publiciste», d'artisan ou d'écrivain. Les «jeunes gens" de Québec constituent donc un milieu plutôt qu'une classe d'âge.

\section{Des «jeunes gens» en lecteurs}

C'est à ces «jeunes gens» comme groupe, solidairement, que s'adresse L'Abeille canadienne que fonde Garneau en 1833, laquelle vise "la diffusion des connaissances, et le goût de la lecture, en variant les sujets ${ }^{40} »$. Explicitement associé au modèle du «magasin»

${ }^{38}$ Sur Napoléon Aubin, voir Jean-Paul Tremblay, À la recherche de Napoléon Aubin, Québec, Presses de l'Université Laval, 1969, coll. «Vie des lettres canadiennes ». Le Fantasque que rédige Aubin possède de grandes qualités littéraires. Voir Lucie Villeneuve, Le "journal-fiction» Le Fantasque de Napoléon Aubin (1837-1845): formes théâtrales et romanesques dans le discours journalistique, thèse de doctorat (études littéraires), Montréal, Université du Québec à Montréal, 2008; Myriam Côté, Les personnages comme maîtres d'ouvre du récit de l'actualité dans le journal Le Fantasque de Napoléon Aubin (1837-1845), mémoire de mâ̂trise (littératures de langue française), Montréal, Université de Montréal, 2015.

${ }^{39}$ D'abord membre des Amateurs typographes d'Aubin, sa réputation comme directeur de troupe est bien établie à Québec. La "grande représentation dramatique» durant laquelle est reprise le 22 janvier 1866 la pièce de Petitclair, précédée de Hariadan Barberousse (mélodrame en trois actes), est d'ailleurs annoncée comme étant placée "sous l'habile direction de M. Joseph Savard».

${ }^{40}$ Sur L'Abeille canadienne et L'Institut, voir François Gallays, «F.-X. Garneau et le journalisme», dans Paul Wyczynski (dir.), François-Xavier Garneau: aspects 


\section{Anmonces Nouvelles.}

SALLE DE MUSIQUE.

CRANDE

Representation Dramatique

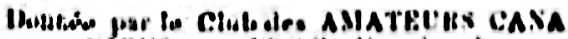

inders, suas l'habilo direstioni de

M. JOBEPH BAVARO

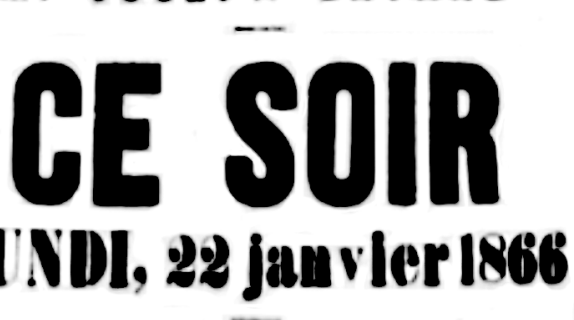

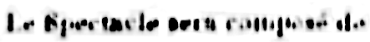

Bibliothèque et Archives nationales du Québec

Figure 2 - Publicité d'une représentation de

la troupe des Amateurs typographes dirigée par

Joseph Savard, Le Canadien, 22 janvier 1866.

- le prospectus évoque Le Magasin pittoresque (créé à Paris en janvier 1833) et le Penny Magazine (créé à Londres en mars 1832) -, l'hebdomadaire est "destiné au peuple, particulièrement à la jeunesse canadienne et aux enfants qui fréquentent les écoles ${ }^{41} »$. Des facilités de paiement sont accordées aux plus pauvres (abonnement payable aux trois mois), et L'Abeille peut être achetée au numéro ${ }^{42}$.

littéraires de son auvre, coll. "Visage des lettres canadiennes», vol. II, Centre de recherches de littérature canadienne-française de l'Université d'Ottawa, Éditions de l'Université d'Ottawa, 1966, p. [47]-63, et Micheline Cambron, «FrançoisXavier Garneau et la presse : écrire, fabriquer et penser le journal», Bulletin d'histoire politique, vol. 27, $\mathrm{n}^{\circ} 1$ (automne 2018), p. 38-65.

${ }^{41}$ L'Abeille canadienne, 7 décembre 1833.

${ }^{42}$ Le prospectus de L'Abeille manifeste une étonnante conscience de classe: «Depuis quelques années, les publications destinées à l'instruction du peuple 
La publication ne dure que 10 numéros, vraisemblablement parce que le nombre d'abonnés n'a pas atteint le millier espéré ${ }^{43}$. Garneau récidive, toujours dans le même esprit, corédigeant avec David Roy l'hebdomadaire L'Institut. Publication scientifique, industrielle et littéraire, à partir du 17 avril 1841, afin de témoigner de son appui au projet de création de l'Institut proposé par Alexandre Vattemare ${ }^{44}$. La tentative fait long feu et le dernier numéro paraît le 22 mai 1841. Encore une fois, il s'agissait de proposer un périodique bon marché pour la bourse dégarnie des étudiants et des ouvriers.

James Huston, qui fut d'abord apprenti typographe et signa quelques textes dans Le Fantasque sous le pseudonyme de l'Apprenti avant d'accéder au statut de typographe et de signer des lettres publiées dans le même Fantasque sous le pseudonyme de l'Arti$\operatorname{san}^{45}$, fonde en octobre 1842 le journal L'Artisan, selon les mêmes principes, se réclamant d'ailleurs des essais de Garneau. Huston mettra fin à l'expérience en juillet 1843, non sans avoir expliqué que tous les abonnés seront remboursés ${ }^{46}$. Peu après, Napoléon

s'accroissent rapidement, surtout en France et en Angleterre. Les avantages qui en sont déjà résultés sont innapréciables $[s i c]$. Jusqu'alors les lumières n’avaient été que le partage des classes riches [...]».

${ }^{43}$ Dans le premier numéro de L'Abeille, le 7 décembre 1833, on apprend que le nombre de 500 souscripteurs sera nécessaire à l'impression du premier numéro. Le rédacteur vise ensuite mille abonnés. Rappelons que Québec compte 22101 habitants en 1825. Yvan Lamonde et Claude Beauchamp, Données statistiques sur l'histoire culturelle du Québec (1760-1900), Chicoutimi, Institut interuniversitaire de recherches sur les populations, 1996, p. 19.

${ }^{44}$ L'Institut succède, en le transformant en profondeur, à l'hebdomadaire Le Journal des étudiants, publié à partir du 12 décembre 1840 .

45 À la suite des premières parutions de «Boîte de Pandore», signées Un apprenti, une brève polémique s'engage hors des pages du périodique autour de l'identité du signataire. Le journal tranche: il ne s'agit pas du pseudonyme d'une personnalité connue et instruite, mais d'un véritable apprenti (Le Fantasque, 7 décembre 1840). Pour un échantillon de l'écriture de l'Artisan, voir l'article "Boîte de Pandore", Le Fantasque, 8 février 1841. L’entrefilet "L'Artisan", dans Le Fantasque du 8 octobre 1842, dévoile indirectement l'identité de l'Artisan en annonçant la publication prochaine du journal.

${ }^{46}$ Le journal reprend en décembre, racheté par Stanislas Drapeau, mais abandonne alors sa mission de journal à bas prix. 
Aubin commence à publier, parallèlement au Fantasque, le journal Le Castor:

Nous aurons pour objet de fournir aux classes de lecteurs les moins aisés un journal qui par la modicité du prix et son contenu soit tout-à-fait à sa portée. Depuis L'Institut qui pour sept shillings et demi par année avait entrepris de publier quatre et parfois six grandes pages de littérature et de science, jusqu’à L'Artisan, qui pour la même somme donnait encore six grandes pages par semaine, les essais n'ont pas manqués $[s i c]$ en ce genre et, nous le savons, nul n'a encore été couronné de succès ${ }^{47}$.

L'intention explicite de ces journaux à bas prix est l'instruction par la lecture, laquelle repose sur le principe de l'autonomie intellectuelle des citoyens. Les tarifs en vigueur et, surtout, la possibilité d'acheter le journal au numéro témoignent nettement de ce désir d'accessibilité du savoir ${ }^{48}$. Toutefois, les quatre périodiques sont fort différents.

L'Abeille canadienne est un "magasin" tourné vers les connaissances utiles et détaché de l'actualité. L'Institut est marqué, lui aussi,

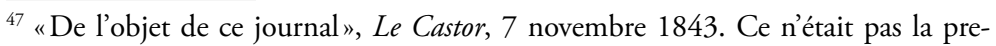
mière tentative d'Aubin. Même si les rédacteurs du journal Le Télégraphe $=$ The Telegraph, Philippe Aubert de Gaspé fils et Napoléon Aubin, n’insistent pas sur le lectorat qu'ils visent, ce trihebdomadaire bilingue, fondé en 1837 et vendu au numéro pour deux sous, se voulait aussi un journal à prix «modique» («LE TÉLÉGRAPHE! Le Télégraphe, Messieurs! Qui veut acheter le Télégraphe? Qui n'achètera pas le Télégraphe? On ne peut se passer du Télégraphe.", Le Télégraphe, 20 mars 1837). L'expérience dure peu. Plus tard, Aubin projettera de publier un " extra ou bulletin" du Fantasque qui serait vendu deux sous et serait ainsi mis à la portée "de la classe peu aisée» (11 août 1838). Aubin répond ainsi à une missive de l'Artisan, qui n'est autre que Huston. L'idée est reprise le 21 septembre 1840. Il s'agirait cette fois de publier le journal deux fois la semaine, le lundi huit pages, le jeudi quatre pages avec des tarifs respectifs de quatre et de deux sous. Il ne semble pas que ces projets se soient matérialisés, mais les collections sont lacunaires.

${ }^{48}$ La vente au numéro, interdite en France, inconnue en Angleterre, apparaît aux États-Unis dans la même décennie (The New York Sun est vendu deux cents en 1833, le New York Herald, deux cents en 1835). Voir Frédéric Charbonneau et Rachel Lauthelier, "Facture et lecture du Canadien", dans Micheline Cambron (dir.), Le Journal Le Canadien: littérature, espace public et utopie (1836-1845), Montréal, Éditions Fides, 1999, p. 96, coll. "Nouvelles études québécoises». 
par un éclectisme certain dans le choix des sujets traités, comme l'illustre son sous-titre: Publication scientifique, industrielle et littéraire. Les textes informatifs sur les villes et les pays étrangers, les découvertes scientifiques ou techniques y côtoient les textes littéraires. Le ton n'est guère unifié: langage technique, formes liées à la vulgarisation et poésie sont juxtaposés dans les pages - ce qui n’est guère différent de la polyphonie des journaux de l'époque. Toutefois, L'Institut comporte des textes d'actualité, livre des "comptes rendus " des séances de la Société littéraire et historique de Québec et de l'Académie des sciences de Paris, témoignant ainsi de sa foi dans le progrès scientifique et du désir de montrer la science qui se fait, se démarquant ainsi nettement de L'Abeille canadienne ${ }^{49}$.

De son côté, L'Artisan de Huston ${ }^{50}$, un bihebdomadaire, est un véritable journal, et non un "magasin». Certes, il poursuit lui aussi des objectifs didactiques, publiant notamment une rubrique sur l'agriculture, qui vulgarise des principes d'agronomie. Mais il affiche en plus, contrairement aux journaux de Garneau, une dimension politique, quoiqu'il se présente comme non assujetti à un parti. Par exemple, l'appel à manifester en faveur de Bagot, lancé par Le Fantasque, reçoit des échos dans L'Artisan, Huston faisant partie de la liste des personnes qui convoquent une assemblée publique "en faveur de l'administration Bagot», le 23 décembre $1842^{51}$. L'«Hommage du Petit Gazetier, aux lecteurs de L'Artisan,

${ }^{49}$ Voir, à propos de ces deux périodiques, Micheline Cambron, «François-Xavier Garneau et la presse: écrire, fabriquer et penser le journal», Bulletin d'histoire politique, vol. 27, $\mathrm{n}^{\circ} 1$ (automne 2018), p. 38-65.

${ }^{50}$ Plusieurs journaux parisiens portèrent ce titre: L'Artisan, journal de la classe ouvrière, en 1830; L'Artisan, journal des classes laborieuses [...], en 1836; L'Artisan, moniteur ouvrier de Paris et des départements, en 1842-1843 (source: Eugène Hatin, "Index", Bibliographie historique et critique de la presse périodique française ou Catalogue systématique et raisonné... précédé d'un essai historique et statistique sur la naissance et les progrès de la presse périodique dans les deux mondes, Paris, Didot, 1866, p. 609).

${ }^{51}$ Le Fantasque, 17 décembre 1842. Font aussi partie de la liste des 144 noms: N. Aubin, P. Chauveau, Ed. Glackemeyer, F.-R. Angers, W. H. Rowen. 
premier jour de l'an $1843^{52}$ ", signé P. Petitclair, porte précisément sur le rôle pacificateur de Bagot ${ }^{53}$. La dimension polyphonique du journal est nette: nombreuses lettres de lecteurs, variété des signatures, invitations de sociétés diverses, feuilletons et aussi présence récurrente du théâtre (plusieurs pièces y sont reproduites, dont, en première page et par livraisons successives les 15, 19, 22, 26 et 29 décembre 1842, La Donation de Pierre Petitclair, placée sous l'en-tête «Littérature canadienne»). Cette polyphonie contribue, au moins autant que l'exercice de la parole dans les sociétés de «jeunes gens", à la théâtralisation de l'espace public ${ }^{54}$.

Quant au Castor, journal politique, littéraire, des arts, de l'agriculture et $d u$ commerce, propriété de Napoléon Aubin et de

${ }^{52}$ L'Artisan, 2 janvier 1843, p. 1; Le Canadien, 4 janvier 1843, p. 2 ; Le Journal de Québec, 10 janvier 1843, p. 2. La feuille volante, en principe remise aux abonnés au Jour de l'An, n'a pas été retrouvée. L'adresse aux destinataires subit de légères modifications selon les journaux: les "lecteurs" de L'Artisan deviennent des "souscripteurs" dans Le Canadien et des "abonnés" dans Le Journal de Québec. Sur les étrennes, voir Micheline Cambron, "Pauvreté et utopie: l'accommodement poétique selon le Petit Gazetier du journal Le Canadien", dans Michel Biron et Pierre Popovic (dir.), Écrire la pauvreté, Actes du VIe Colloque international de sociocritique, Université de Montréal, septembre 1993, Toronto, Éditions du GREF, 1996, p. 301-317.

${ }^{53}$ Le timbre n'est pas donné, mais comme ces étrennes sont signées Pierre Petitclair, compositeur dont plusieurs pièces musicales sont parues dans Le Ménestrel, la mélodie est vraisemblablement de lui. Le texte est reproduit, sous le titre "Le règne du juste", privé de la strophe de demande d'obole du petit gazetier qui le terminait originellement, dans Le répertoire national de Huston, t. II, p. 306-307.

${ }^{54}$ Yvan Lamonde relève combien la presse est vue par la jeunesse comme ce que Joseph Doutre qualifie de "seul théâtre» qu'elle peut alors atteindre (Lamonde, Gens de parole, p. 31-33). Sur cette théâtralisation par l'intermédiaire des journaux, voir Louise Frappier, "Un théâtre de papier: critique et parole dramatique dans Le Fantasque (1837-1846)", dans Marie-Ėve Thérenty et Alain Vaillant (dir.), Presse, nations et mondialisation au XIX siècle, Paris, Nouveau Monde, 2010, p. [263]-275. Soulignons que le premier article de la Revue canadienne naissante est aussi une courte pièce, "Le Jour de l'An ou Les petits cadeaux entretiennent l'amitié» (p. 1-3). Sur le théâtre de la presse, voir aussi Corinne SaminadayarPerrin, Les discours du journal: rhétorique et médias au XIX siècle (1836-1885), Université de Saint-Étienne, 2007, 269 p., en particulier «L'éloquence en spectacle», p. 43-68. 
W. H. Rowen ${ }^{55}$, bihebdomadaire lui aussi, il se présente comme un journal plus politique que culturel, défendant «la conservation de la nationalité française en ce pays» et promouvant la discussion "par la voix populaire». L'une des mesures qu'il appuie est « une bonne loi de l'enseignement primaire ${ }^{56} »$. Il recèle une masse exceptionnelle d'informations sur la vie associative de Québec, entre autres, un calendrier des "assemblées de sociétés ». Le journal mourra après le second grand incendie de Québec, en juin 1845.

Il me semble qu'il faut voir dans ces tentatives répétées le désir de prendre le relais, d'une manière originale, de la déclaration de principe d'Étienne Parent, qui écrivait au moment de la renaissance du journal Le Canadien, en 1831, que, "de toutes les presses, la presse périodique est celle qui convient le mieux au peuple, c'est de fait la seule bibliothèque du peuple», et affirmait: "[L]e savoir est une puissance. Chaque nouveau lecteur ajoute à la force populaire ${ }^{57}$.» La succession des journaux à bas prix à Québec témoigne du désir continu de créer une communauté de lecteurs qui soit plus étendue et plus diverse de manière à favoriser la discussion démocratique. Les enjeux de l'instruction sont aussi littéraires. Dans la livraison du Castor du 11 juillet 1844, un texte explique le lent développement de la littérature nationale par le manque de lecteurs:

${ }_{55}$ Imprimeur du Fantasque à partir du 23 mars 1839 (Tremblay, À la recherche de Napoléon Aubin, p. [75]), William Henry Rowen fait partie des membres fondateurs de la Société canadienne d'études littéraires et scientifiques (Ibid., p. 140). Selon Darveau, Rowen était membre de la troupe des Amateurs typographes dirigée par Aubin: "Les noms des Savard, des Bélanger, des Bédard, des Rowen, des Vézina, des Sauvageau, des Chartrain, des Jacquies, etc., etc., sont encore vivaces dans la mémoire de ceux qui furent témoins de leurs triomphes scéniques" (Louis-Michel Darveau, Nos hommes de lettres, Montréal, 1873, A. A. Stevenson, p. 8). Il fut en outre l'imprimeur des statuts de la Société SaintJean-Baptiste de Québec et de ceux de la Société canadienne d'études littéraires et scientifiques. Il sera en 1852 l'imprimeur du premier Catalogue de la bibliothèque de l'Institut Canadien.

${ }^{56}$ Les extraits cités sont tirés de «De l'objet de ce journal ", Le Castor, 7 novembre 1843 , p. 2.

57 "Adresse au public canadien", Le Canadien, 7 mai 1831, p. 1. 
Il est évident que jusqu'à présent, nous n'avons que très-peu [sic] de choses à montrer à celui qui voudrait juger de notre pays, de nos mœurs, de nos progrès sans venir habiter quelques années parmi nous. La cause de cette espèce de disette bibliographique peut s'expliquer autrement que par une disette d'auteurs; jusqu'à présent, il faut le dire, on a manqué de lecteurs; la population lettrée n'a pas été assez considérable pour fournir un public et celui qui aurait mis au jour une œuvre d'imagination n'en eût pas été quitte pour ses frais d'impression: les sarcasmes seraient venus s'ajouter à l'amertume de ses déboires. Désormais, il n'en sera plus ainsi : les établissements d'éducation qui florissent [sic] dans toutes les parties du pays versent chaque année dans la société une population nouvelle et nombreuse [...]. Voilà l'ère nouvelle que la génération qui prend la place de celle qui passe doit surtout comprendre et faire naître; voilà l'œuvre à laquelle chacun de nous doit prêter la main, les uns par le travail, les autres par l'encouragement.

Cette analyse se termine par l'évocation de deux projets en cours: celui de François-Xavier Garneau, qui rédige alors son Histoire $d u$ Canada, et celui de Pierre-Joseph-Olivier Chauveau, qui lance une souscription pour son Essai sur la littérature française dont l'appel à souscriptions, déposé, entre autres, chez «l'éditeur, $\mathrm{M}^{\mathrm{r}}$ Aubin» apparaît sur la même page (figure 3). D'où il faut conclure que, si les journaux à bas prix accroissent le nombre de leurs lecteurs, ils constituent aussi une étape importante vers la réalisation de travaux intellectuels et littéraires d'envergure.

La brève histoire des journaux à bas prix que nous venons d'esquisser met aussi en relief le caractère égalitaire de cette communauté de lecteurs. Les fondateurs de ces journaux sont jeunes, et plusieurs sont autodidactes. Ils publient d'abord pour leurs pairs, ce qui leur paraît utile, voire nécessaire au progrès de l'esprit. Aux lecteurs de juger et de transmettre. 
Sous t.E l'ATRoxage

D):

\section{L'Associalion de la Biblialhèque dé Québcc.}

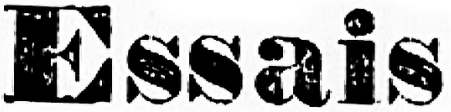

\section{Sur l'Elat de la Lilteraturo en France}

Depuis la rivolution de $17 \mathrm{S9}$.

CET ourrage comprendra la lechre donnce par Mr. Cha $\checkmark$ daus le tnois de Suin bernier qui seru considérnlylement sugunentev, et In leciure que ce Mousieur se propose de doniler dans le mois de: Steptembre prochatin.

Il y scra ajouté une table alphobétique de auteurs nommés dạns le cours de lourrage, aree une courle notice hiographique et bibliuarnpligute sur chacun d"eux, at des extrates contenant l'opintion des critipues lo- plus éninents tels que Ladane, Chenan,

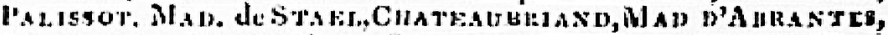

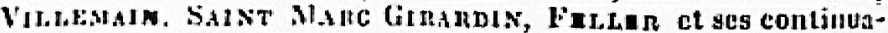

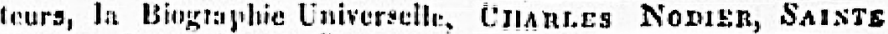

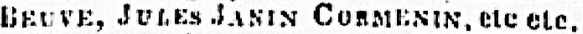

J."ouvrage formerd un lieau volume in 80. ou grand is 120,

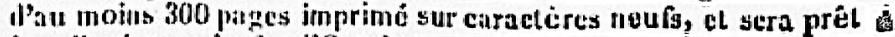
Cetre lisré vern la tin d'Oclobre.

Le prix seru j'UxE PLAstae powr les souscripteats.

On sonferil chez] éliteur, Mr. At us, burea u du Cirsor, Itho Couillart 14, et dies listes de souscription hont aussi dépuséc a a tax entroils suivanls :

Au Salonde Latecture de l'Ass cialion de la l'ilitipt bünne de Quehec. au lurea du Journal du Quéliece ä la tihrainio de Mr iró. cliette rus: Lamontapne, it celle de Mesars Cary $\$$ Cic, rue Buade et it la Iibratire Ecelcsiastique, rue Ste. Fomilte.

AGexts. Montreal, E. R. Fubre l*r. lihraire.

Trois Riw. D. It. Myrand, Her. Dê.- Rérist.

Bylown at le Haut C'amath, Ed, Màsse Eer.

Quéliec 11 Juillet 1844.

Figure 3 - Publicité de la souscription pour le livre de Chauveau, Le Castor, 11 juillet 1844. 


\section{Des « jeunes gens» en écrivains...}

Ces périodiques invitent aussi leurs lecteurs à écrire. Ceux qui le font, dès le milieu des années trente, ont de l'ambition: écrire des étrennes sur une musique originale (Angers et Aubin, 1836 ${ }^{58}$ ) (figure 4), revendiquer la traduction d'un poème (Angers, 1837 ${ }^{59}$ ), faire paraître le premier roman de mœurs (de Gaspé, $1837^{60}$ ), publier la première pièce de théâtre écrite par un Canadien de naissance (Petitclair, 183761). Ils seront plus ambitieux encore dans les années 1840: première tragédie (Le jeune Latour, GérinLajoie, $1844^{62}$ ); première histoire du Canada depuis sa fondation, écrite d'un point de vue canadien, et en plusieurs tomes (Histoire du Canada depuis sa découverte jusqu'à nos jours, Garneau, 1845-1848); édition du premier répertoire d'œuvres canadiennes publiées dans les journaux, livré «au jugement de ceux qui voudront en faire l'étude", en quatre volumes (Le répertoire national, Huston, 1848-1850 ${ }^{63}$ ).

58 «Hommage du Petit Gazetier à Messieurs les abonnés du Canadien, le premier jour de l'année 1836 ", chant patriotique du Canada, paroles de M. F. R. A. [François-Réal Angers] et musique de M. N. [Napoléon] Aubin, feuille volante. Source (avec titre erroné, mais reproduction du fac-similé de la feuille volante): Maria Calderici-Bryce, "La politique de l'édition musicale au Canada avant 1867 ", Les Cahiers de l'Association pour l'avancement de la recherche en musique au Québec, n 6, 1985, p. 2-13.

${ }^{59}$ Angers signe le 14 avril une correspondance dans laquelle il se déclare l'auteur de la traduction du poème de Moore, "Canadian Boat Song", parue dans le même journal le 12 avril. "Correspondance», Le Télégraphe, 14 avril 1837.

${ }^{60}$ Annonce de la publication $\mathrm{du}$ "[r] oman historique L'Influence d'un livre», qui "paraîtra au commencement de MAI prochain", Ibid.

${ }^{61}$ "Comédie. Prospectus», [Appel à souscriptions pour "Qui trop embrasse mal étreint»], Le Canadien, 7 novembre 1836.

${ }^{62}$ La pièce est d'abord jouée au collège de Nicolet en juillet 1844, puis publiée quasi simultanément dans trois périodiques en septembre 1844. Voir Isabelle Décarie et Louise Frappier, "La tragédie du Jeune Latour dans Le Canadien: interférences textuelles et politiques», Études françaises, vol. 36, n 3 (2000), p. 27-45.

${ }^{63}$ Remarquons que cette première édition est imprimée par Napoléon Aubin, rédacteur du Télégraphe, du Fantasque et du Castor. 


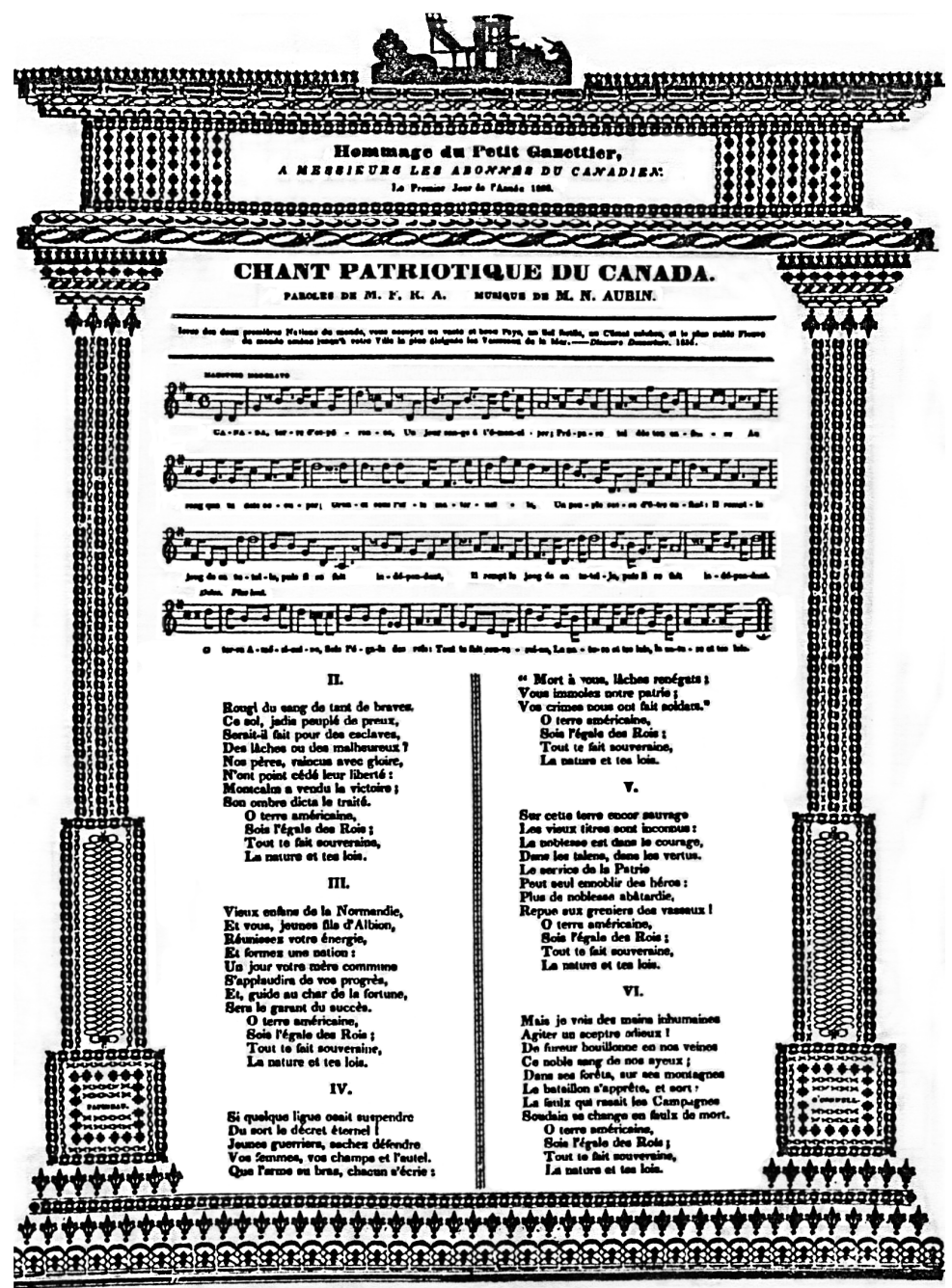

Figure 4 - «Étrennes du Canadien», 1836.

Les œuvres de la période, principalement écrites par des jeunes gens, remarquons-le ${ }^{64}$, ne sont pas seulement ambitieuses, elles

${ }^{64}$ Rappelons que Garneau, l'un des aînés, n'a que 36 ans au moment où il publie le premier tome de son Histoire du Canada. 
sont traversées par un postulat: l'importance de l'instruction. Les romans de Pierre-Joseph-Olivier Chauveau et d'Antoine GérinLajoie ont souvent été abordés comme des romans d'apprentissage dans lesquels la formation des jeunes gens se trouve thématisée et donnée comme un trait du parcours romanesque des personnages. Mais la question des savoirs y joue un rôle autrement plus considérable. Pensons aux discussions sur la propagation du choléra dans le premier et aux enseignements pratiques sur l'agriculture, l'industrie, la bibliothéconomie du second ${ }^{65}$. La question de l'éducation, dont la présence médiatique massive a été relevée et analysée pour la décennie 1830 dans l'ouvrage de Jean-Pierre Proulx ${ }^{66}$, ne se réduit donc pas à des débats théoriques, la littérature y prend part aussi. Deux exemples me paraissent particulièrement éloquents.

Dans le roman de Joseph Doutre, Les fiancés de $1812^{67}$, qui paraît en 1844 , les personnages féminins sont importants. Ils se distinguent par leur éducation, et une partie de leur charme tient à leur savoir. Ithona, la jeune Amérindienne qui a sauvé la vie du héros, Alphonse, est confiée par un Alphonse indifférent à une maison d'éducation; son instruction est déterminante puisque, grâce à elle, Alphonse "plie enfin invinciblement sous les charmes agrestes de cette fleur des bois ${ }^{68}$ ". Louise, l'héroïne, se tire quant à elle d'un fort mauvais pas parce qu'elle parle «l'anglais avec beaucoup de facilité. Elle [a] reçu son

${ }^{65}$ Les débats mis dans la bouche des personnages par Chauveau sont étonnamment proches de ceux que l'histoire de la médecine nous a restitués. Voir Denis Goulet et André Paradis, Trois siècles d'histoire médicale au Québec: chronologie des institutions et des pratiques (1639-1939), Montréal, VLB éditeur, 1992. Sur les savoirs dans le roman de Gérin-Lajoie, voir Micheline Cambron, "Les bibliothèques d'Antoine Gérin-Lajoie", Études françaises, vol. 28, nº 4 (1993), p. 135-150.

${ }^{66}$ Voir, en particulier, le chapitre "Les méthodes d'enseignement», qui fait fond sur une riche documentation, soit plus de cent articles publiés dans les périodiques francophones, sans compter les autres documents (Proulx et al., La genèse de l'école publique et de la démocratie scolaire au Québec, p. 305-352).

${ }^{67}$ Joseph Doutre, Les fiancés de 1812: essai de littérature canadienne, Montréal, Louis Perrault, imprimeur, 1844. Édition en fac-similé par Réédition-Québec, 1969.

${ }^{68}$ Ibid., p. 266. 
éducation en partie de dames anglaises qui avaient établi à Montréal une académie très renommée ${ }^{69}$ \%. Retenons de ces exemples que les femmes ne perdent pas leur temps lorsqu'elles s'instruisent: elles développent des armes de séduction, mais aussi de défense fort utiles ${ }^{70}$.

Les effets de l'éducation ne sont pas moins déterminants dans le roman La terre paternelle de Patrice Lacombe, publié anonymement sous la rubrique «Études de mœurs» de L'Album littéraire et musical de la Revue canadienne en février 1846. Le narrateur intervient, dès les premières pages, pour regretter que seule la fille de la famille Chauvin sache lire, écrire et compter:

Les parents, par une coupable indifférence, avaient entièrement négligé l'éducation de leurs garçons [...] tout avait été fait pour le cœur, rien pour l'esprit. Marguerite là-dessus avait l'avantage sur ses frères. On l'avait envoyée passer quelque temps dans un pensionnat où le germe des plus heureuses dispositions s'était développé en elle; aussi c'était à elle qu'était dévolu, chaque soir, après le souper, le soin de faire la lecture en famille; les petites transactions, les états de recette et de dépense, les lettres à écrire et les réponses à faire, tout cela était de son ressort et lui passait par les mains, et elle s'en acquittait à merveille ${ }^{71}$.

Ce jugement possède un poids considérable dans un si court roman. Lacombe fait de Marguerite le seul personnage qui lit et auquel, donc, peut s'identifier le lecteur. Dans la scène de la donation, celle-ci pouffe d'ailleurs, comme le lecteur, devant le ridicule du "cochon raisonnable»:

- Tenez, s'écria-t-il, je m'en vais vous mettre d'accord; vous, père Chauvin, vous exigez un cochon gras; vous, le fils, vous trouvez que c'est trop fort; hé bien, mettons:

${ }^{69}$ Ibid., p. 187.

${ }^{70}$ Sur l'instruction dispensée aux femmes par des laïques, voir Andrée Dufour, "Les premières enseignantes laïques au Québec: le cas de Montréal, 18251835", Histoire de l'éducation, no 109 (2006), [En ligne], [http://histoire-education.revues.org/1326] (11 mars 2017).

${ }^{71}$ Patrice Lacombe, La terre paternelle, Montréal, BQ, 1993, p. 29. 
- Un cochon raisonnable.

- C'est cela, c'est cela, dirent ensemble tous les assistants.

En même temps, un éclat de rire, mais étouffé presque aussitôt, fit tourner tous les yeux du côté de Marguerite qui, depuis longtemps, faisait tous ses efforts pour se contenir.

Le notaire la regarda, en fronçant légèrement les sourcils:

- Mam'selle, dit-il, pourrais-je savoir le sujet de...?

- Chut! Marguerite, dit le père ${ }^{72}$.

On peut croire que, si elle avait eu à parapher le contrat de donation (ce qu' elle ne fait pas, alors qu'elle est la seule à savoir signer), elle eût pu intervenir et dénoncer l'abus de procédure et la transformation du lien filial qui en résultait, de sorte que la catastrophe eût peut-être été évitée à la famille Chauvin.

Rappelons, par ailleurs, l'importance de l'instruction dans Linfluence d'un livre, démontrée par l'absurde par l'intermédiaire de l'ignorance de Charles Amand, convaincu que sa fortune tient à un livre et envieux de ceux qui sont instruits. De même, le destin des personnages des Révélations du crime de François-Réal Angers est expliqué à l'aide d'une argumentation antinomique qui fait de la prison une école du crime ${ }^{73}$. Enfin, la maîtrise de la langue et du raisonnement acquise au couvent par les personnages de la pièce $L a$ donation de Pierre Petitclair assure le triomphe des jeunes filles ${ }^{74}$. Dans ces œuvres importantes des décennies 1830 et 1840, l'évolution de la diégèse tient aux bons ou aux mauvais savoirs acquis dans ou hors l'école. En somme, l'instruction est dotée dans les textes littéraires de vertus pratiques, finesse du langage et du raisonnement, débrouillardise, qui infléchissent l'issue de la diégèse. Les énoncés qui concernent les pratiques associées aux savoirs jouissent d'une forte performativité, c'est-à-dire qu’ils réalisent dans la fiction ce qu’ils énoncent.

${ }^{72}$ Ibid., p. 48-49.

${ }^{73}$ Les révélations du crime ou Cambray et ses complices: chroniques canadiennes de 1834, présentation et bibliographie par Gilles Dorion, Montréal, Éditions Nota bene, 2003 [1837].

${ }^{74}$ Pierre Petitclair, La donation. James Huston la reproduit sans modification majeure dans Le répertoire national, t. II, p. 234-270. 


\section{... et en professeurs}

Les «jeunes gens» écrivent, ils donnent du poids à leurs essais en les publiant, en les inscrivant dans l'espace public. Ils s'enseignent ainsi les uns aux autres.

C'est précisément la constitution d'un espace public véritable qui est au cœur du projet de LeTourneux et de sa Revue canadienne, fondée en décembre 1844. Les collaborateurs canadiens qui nous sont connus $^{75}$ sont souvent très jeunes: Antoine Gérin-Lajoie, Hector Fabre, Alphonse Poitras, Guillaume Lévesque, entre autres. Les textes ont le plus souvent une portée didactique (même si cela peut être assaisonné d'humour). Et la dimension instructive se trouve réfléchie, dans les dix premiers numéros, par une chronique régulière, signée $M .{ }^{76}$, qui porte sur l'éducation, discute des méthodes, des outils, des principes et même du rôle des parents. L'accès au savoir et la maitrise du jugement sont ici primordiaux. La rubrique «Histoire de la semaine» relate ce qui mérite, dans la vie montréalaise de la semaine, d'être raconté et soumis au jugement éventuellement moqueur du chroniqueur (possiblement Hector Fabre) et des lecteurs. La façon dont la revue se présente dans l'espace public témoigne d'ailleurs de ce désir d'instruire: par son «Prospectus" et par la dédicace inaugurale à la Société des amis ${ }^{77}$; par la page

${ }^{75}$ Les textes des journaux et des revues ne sont pas toujours signés (les poèmes font exception et le sont presque toujours), il y a des pseudonymes. Dans le premier numéro de la Revue canadienne, seuls le "Prospectus» (LeTourneux) et le texte de réception de Guillaume Lévesque à la Société des amis sont signés (Archives du CRILCQ, Université de Montréal).

${ }^{76}$ Possiblement, le juge Mondelet, qui donne une conférence sur l'éducation durant cette période (Revue canadienne, 15 février 1845).

${ }_{77}$ Cette dédicace de Louis-Octave LeTourneux a été reliée, non paginée, en ouverture du premier volume de la revue. Elle explicite ses objectifs: «Il est beau de nous voir réunis tous ensemble par les liens de la plus étroite amitié, tendant vers le grand but de notre perfectionnement moral et intellectuel, et portant tous dans nos cœurs les mêmes espérances pour le bonheur futur de notre commune patrie; peut-être pourrons-nous la servir un jour. Amassons donc les trésors de l'intelligence, ils nous rendront capables de lui être utiles.» 
couverture de l'Album littéraire et musical de la Revue canadienne, qui porte, dès le premier numéro du volume 2 , les quatre mots d'ordre: honneur, éducation, industrie, patrie ${ }^{78}$; par l'incorporation d'un public féminin, explicitée dans la sollicitation des annonceurs: l' "Avis au commerce», placé sur la $4^{\mathrm{e}}$ de couverture, affirme: "Toutes les dames le lisent et ce sont les dames qui font les achats pour les besoins de la famille ${ }^{79}$ " (figures 5 et 6 ).

Le désir d'inscrire les savoirs dans un espace public élargi, qui s'en trouverait ainsi enrichi et affermi, mais aussi de faire œuvre en enseignant à tous était déjà lisible dans les deux projets que Le Castor évoquait en juillet 1844 : l' Histoire du Canada de Garneau et l'Essai sur la littérature française de Chauveau, qui ne se réalisera pas ${ }^{80}$. L’ «ère nouvelle» dont ces projets témoignent est bien celle que « la génération qui prend la place de celle qui passe doit surtout comprendre et faire naître ${ }^{81} »$. Cette génération, c'est donc celle qui va de Garneau à Chauveau et aussi celle de celui qui tient la plume - Aubin, ou Huston, encore à Québec -, celle des jeunes gens de la Société des amis et de l'Institut canadien naissants. L'«œuvre» à laquelle tous doivent participer, selon le mot du Castor, repose sur leur jugement. Ce mot est cardinal dans l'œuvre de Garneau ${ }^{82}$. Le

${ }^{78} \mathrm{La}$ couverture de papier coloré des numéros ne figure pas dans les exemplaires reliés, malheureusement, ni dans les numérisations. Nous perdons ainsi l'image de marque de la Revue et de l'Album ainsi que les publicités qui se trouvent sur ces quatre pages.

${ }_{79} 4^{\mathrm{e}}$ de couverture, l'Album littéraire et musical de la Revue canadienne, juillet 1846 (Archives du CRILCQ, Université de Montréal).

${ }^{80}$ Sur cet ouvrage, voir Lucie Robert, «Germaine de Staël, aux origines de l'histoire littéraire au Québec", dans Martin Doré et Doris Jakubec (dir.), Deux littératures francophones en dialogue: du Québec et de la Suisse romande, Québec, Presses de l'Université Laval, 2004, p. 221-232, particulièrement les pages 223-226. Ce texte est cité dans l'article de Lucie Robert, qui paraît dans le présent numéro, "Les correspondances de Chauveau au Courrier des Etats-Unis, 1841-1849».

${ }^{81}$ Le Castor, 11 juillet 1844.

${ }^{82}$ Micheline Cambron, «Le mystère François-Xavier Garneau: à la recherche du mythe", dans Benoît Melançon et Pierre Popovic (dir.), Miscellanées en l'honneur de Gilles Marcotte, Montréal, Éditions Fides, 1995, p. 345-356. 


\section{LA REGOU TMUDENE.

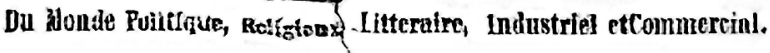

Ce Journal dont la circuation s'étend de jour en jour en

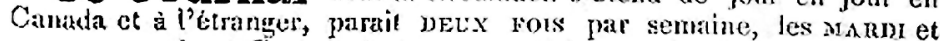
vexdrem-soirs. Il appartient par sa politique an parti porrulaire et à l'oppasition libèrale, et aussi ì ce grand et sublime progrès qui entmine les nations modernes dans lic voie de la liberté commerciale vers la paix universelle et l'union entre tous les peuples.

\section{LOUIS O. Le TOURNEUX, Eeditezr-Réllacteur en Chef.}

Figure 5 - Définition de la Revue canadienne, $2^{\mathrm{e}}$ de couverture, Album littéraire et musical de la Revue canadienne, janvier 1847.

\section{JOURNAL D'ANNONCES DES DAIIES.}

\section{AVIS AU COMMERCE.}

N nous a engagé à mettre sur le couvert de notre ALBUN des annonces, qui intéressent les Dames et les familles, tels que les grands Hotels, les maisons et les magasins le plus en vogue dans la Capitale. Nous nous empressons de nous réndre à ce dósir, dans l'intérêt du commerce Canadien.

Les marchands doivent comprendre que par sa nature même, notre Album offre au commerce la plus grande voie de publicité, car c'est un fitit connu que cette publication mensuelle a déjà plus de circulition dans ce pays qu'aucune feuille qualconque. Il n'y a pas une famille où J'ALBUM ne pénètre d'une manière ou d'une autre. Vous le rencontrez dans tous les salons. Toutes les Dames le lisent et ce sont les Da-

mes qui font les achats pour les besoins de la famille.
Ainsi désormais les annonces de l'A LBUM de la Revue Canadienne sont indispensables à toutes les industries qui se rattochent de près on de loin, aux besoins générnux de la famille, mais surtot. à la toilette des Dames et à la vie élégante, telles que les magasins de nouvcautés, modistcs lingères, Tournisseur's, magasins de dentelles, gants, musicue, livres, cliapeaux, menbles, bijoux, fourrures, tapis, toiles cirées, \&c., \&ce. RT Los annonces sont recues aux Bureaux de la Revue Canmlicnne, No. 15, Rue St. Vincent. Prix des annonces do l'Album; 20 chelins par année pour un quarré de 12 lignes, d'une seule colonne, lo double pour deux colonnes, et ainsi de suite.

Figure 6 - «Avis au commerce», $4^{\mathrm{e}}$ de couverture, Album littéraire et musical de la Revue canadienne, juillet 1846.

projet de Chauveau est de même nature: «[I]l a exercé son jugement», affirme le rédacteur ${ }^{83}$. Remarquons qu'ici le savoir n'est pas rapporté à des autorités, mais à l'exercice du jugement de celui qui écrit. Un savoir démocratique, en somme. Nous sommes au plus près des idéaux qui marquaient le désir de regroupement et

${ }^{83}$ Le Castor, 11 juillet 1844. 
d'instruction mutuelle des "jeunes gens». Sans doute ces idéaux sont-ils en partie puisés dans les œuvres philosophiques et politiques françaises de la période ${ }^{84}$. Mais ils trouvent probablement aussi leur source dans la fréquentation d'écoles inspirées par les méthodes d'enseignement mutuel ou la fréquentation de ceux qui furent marqués par ces pratiques pédagogiques, dont le sens politique est ravivé par les nombreux débats sur l'éducation auxquels ont participé et participent les "jeunes gens», dont certains sont déjà moins jeunes.

\section{Une vision mutualiste et démocratique de l'éducation}

L'ensemble des remarques qui précèdent gagnerait à être placé de manière plus explicite en relation avec les valeurs de l'enseignement mutuel, enseignement dont il faut rappeler qu'il sera graduellement écarté au même moment en France, même si, à l'origine, Guizot en était un farouche partisan, au profit d'un enseignement simultané dans lequel le maître retrouve la place centrale ${ }^{85}$. Mais, en l'état, il me semble avoir démontré que les années 1840 sont, dans la foulée des années 1830, durant lesquelles l'éducation est l'une des plus vives préoccupations qui traversent le discours culturel, marquées par une conception mutualiste et démocratique de l'éducation, terme qui renvoie non seulement à un enseignement primaire populaire, mais aussi au perfectionnement des jeunes (et des moins jeunes) gens.

Concluons rapidement en indiquant quelques hypothèses qui paraissent devoir être examinées sur la base de ce bilan.

${ }^{84}$ On peut penser à Guizot, bien sûr, mais aussi à la conviction en l'éducation qui se trouvera cristallisée, à peine plus tardivement, dans le discours de Victor Hugo du 11 novembre 1848 ("Discours à l'Assemblée nationale [1848-1871]", Assemblée nationale, 11 novembre 1848, [En ligne], [http://www.assembleenationale.fr/histoire/victor_hugo/discours_fichiers/seance_11 novembre 1848 . asp] (3 mars 2018)). Je remercie Mathilde Cambron-Goulet de m'avoir rappelé l'importance de ce texte cardinal.

${ }^{85}$ Voir Nique, Comment l'école devint une affaire d'État (1815-1840), et Jouan, "Enseignement mutuel et enseignement simultané». 
La première est la dimension performative qui traverse tant les discours sur l'éducation que ceux sur la littérature: l'instruction apparaît comme un facteur de changement social, un vecteur de réduction des écarts sociaux, un outil de maîtrise du monde, par la lecture, l'écriture et l'enseignement. Il faudrait lire, relire les textes de ces «jeunes gens» pour en dégager non seulement l'ethos utopique, mais aussi une conception du langage qui place la discussion au cœur des changements sociaux. Cela a, bien sûr, des effets sur la définition de la littérature qui a alors cours, mais aussi sur la dimension démocratique qui l'imprègne.

La seconde est plus hardie et me vient de la lecture des Réformistes d'Éric Bédard ${ }^{86}$. Je crois qu'il faut faire l'hypothèse que l'épisode de l'éviction de James Huston de la présidence de l'Institut canadien n'est pas seulement lié à une rupture politique, mais tient aussi à la minorisation des jeunes gens, qui sont autodidactes ou sont des "étudiants de première génération", comme on dit aujourd'hui, ceux dont le salut intellectuel est venu de l'école primaire en quelque sorte. Bédard a raison de faire remarquer que les "réformistes» sont allés dans les collèges, mais j'ajouterai qu'ils viennent majoritairement de familles dans lesquelles l'accès à l'éducation était "naturel», allait de soi. Il faudrait examiner de plus près la trajectoire intellectuelle des jeunes gens, dont il me semble qu'ils forment de fait deux groupes: l'un pour lequel l'instruction est un donné, l'autre pour lequel l'instruction est un acquis. La polarisation des forces qui marque le Québec des années 1845-1850 se caractérise au moins autant par la tension entre des rapports différenciés au savoir et à l'éducation, que par une fracture de nature politique partisane.

Enfin, le repli sur la culture qui marquerait, selon Dumont, le mouvement des esprits durant le régime de l'Union, gagnerait sans doute à être abordé du point de vue des autodidactes, comme

${ }^{86}$ Éric Bédard, Les réformistes: une génération canadienne-française au milieu du $X I X^{e}$ siècle, Boréal compact, 2012. Bédard insiste sur les débats autour de la question de l'éducation, à de nombreux moments de son argumentation. 
Garneau et Huston, qui sont, de fait, les deux seuls ${ }^{87}$ qui, avec le dramaturge Pierre Petitclair, issu de l'enseignement mutuel, produiront une œuvre d'envergure et "enseigneront» à leurs descendants en les invitant à exercer leur jugement.

${ }^{87}$ Rappelons que le projet intellectuel original et ambitieux de Pierre-JosephOlivier Chauveau ne verra pas le jour. Quant au Répertoire de Huston, il s'agit d'un travail exceptionnel, qui repose sur des recherches documentaires, un protocole d'édition explicité et une maîtrise typographique remarquable. Voir Cambron, " "Place aux textes!" : littérature québécoise et injonctions de lecture». 